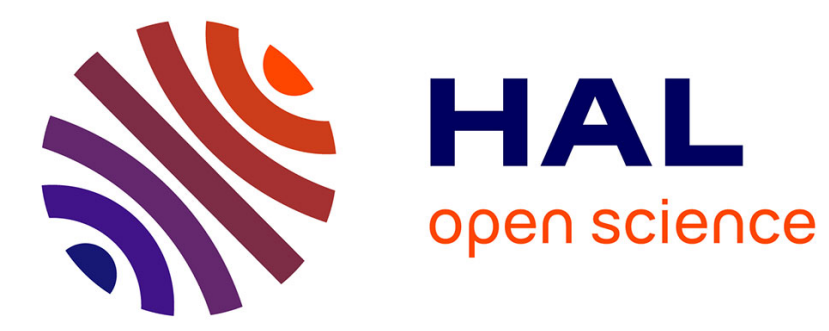

\title{
Orthogonal polynomials or wavelet analysis for mechanical system direct identification
}

\author{
Corinne Rouby, Didier Rémond, Pierre Argoul
}

\section{To cite this version:}

Corinne Rouby, Didier Rémond, Pierre Argoul. Orthogonal polynomials or wavelet analysis for mechanical system direct identification. Annals of Solid and Structural Mechanics, 2010, 1 (1), pp.41-58. 10.1007/s12356-009-0005-1 . hal-00485149

\section{HAL Id: hal-00485149 \\ https://hal.science/hal-00485149}

Submitted on 29 May 2019

HAL is a multi-disciplinary open access archive for the deposit and dissemination of scientific research documents, whether they are published or not. The documents may come from teaching and research institutions in France or abroad, or from public or private research centers.
L'archive ouverte pluridisciplinaire HAL, est destinée au dépôt et à la diffusion de documents scientifiques de niveau recherche, publiés ou non, émanant des établissements d'enseignement et de recherche français ou étrangers, des laboratoires publics ou privés.

$$
\text { Copyright }
$$




\title{
Orthogonal polynomials or wavelet analysis for mechanical system direct identification
}

\author{
C. Rouby · D. Rémond · P. Argoul
}

Received: date / Accepted: date

\section{Abstract Keywords}

\section{Introduction}

Parameter identification is a fundamental problem in structural mechanics. Identification techniques can be based on the modal parameters through the dynamic responses of the structure and are then qualified as indirect, or on the general matrix equation of dynamic equilibrium and are then qualified as direct.

In direct approches, orthogonal functions are frequently used because of their integration property, based on a square matrix with constant elements. This property allow to transform the set of differential equations which governs the dynamical behaviour of the system into a set of algebraic equations. Pacheco and Steffen (2002) compare this technique with different kinds of orthogonal functions, such as Fourier series, Legendre polynomials, Jacobi polynomials, Chebyshev polynomials, Block-Pulse functions and Walsh functions. Rémond et al (2008) develop this method, using the Chebyshev polynomials and dissociating signal expansions and parameter estimation. They proposed indeed to identify parameters by using only a few points from the acquired data.

Wavelet analysis can also be used for modal identification. Staszewski (1997) apply the coutinous wavelet transform to the free vibratory response of a mechanical system to estimate its damping, considering the Morlet wavelet function. Slavic et al (2003) proposed a closely related method, using the Gabor wavelet function. A complete procedure for modal identification from free responses based on the continuous wavelet transform is presented by Le and Argoul (2004), comparing characteristics of Morlet wavelet, Cauchy wavelet and harmonic wavelet. These techniques have also been

C. Rouby · D. Rémond

Université de Lyon, CNRS UMR5259, LaMCoS, INSA-Lyon, F-69621, Villeurbanne, France E-mail: corinne.rouby@insa-lyon.fr, didier.remond@insa-lyon.fr

C. Rouby · P. Argoul

Université Paris-Est, UR Navier, École des Ponts ParisTech, 6-8 av Blaise Pascal, Cité Descartes, Champs sur Marne, 77455 Marne la Vallée Cedex 2, France E-mail: rouby@lami.enpc.fr, pierre.argoul@lami.enpc.fr 
applied to free responses of linear non-proportionally damped systems (Erlicher and Argoul, 2007) and to weakly non-linear systems Staszewski (1998).

The aim of this paper is to propose a direct method of identification, transforming a set of differential equations into a set of algebraic equations, based on either orthogonal polynomials or wavelet analysis. Whereas classical identification methods using wavelet analysis applied to free responses, forced systems are here considered. The general scheme of the proposed technique is presented in $\S 2$. The case of identification using orthogonal polynomials is studied in $\_3$, the chosen basis being the one of Chebyshev, and the case of identification using wavelet analysis is studied in $\S 4$, the chosen wavelet mother being the Cauchy wavelet. In $\S 5$, both methods are tested on numerical simulations of a three degrees of freedom system.

\section{Description of the unified identification method}

The expression of the equation of motion for a multi Degrees of Freedom (DoF) linear system can be written in the form

$$
\underline{\underline{M}} \underline{\ddot{x}}(t)+\underline{\underline{C}} \underline{\dot{x}}(t)+\underline{\underline{K}} \underline{x}(t)=\underline{f}(t),
$$

where $\underline{x}$ (resp. $\underline{\dot{x}}, \underline{\ddot{x}}$ ) refers to the vector of size $k$ of the mass displacements (resp. velocity, acceleration), $f$ refers to the vector of size $k$ of forces applied on the system, and $\underline{\underline{M}}$ (resp. $\underline{\underline{C}}, \underline{\underline{K}}$ ) refers to the $k \times k$ mass (resp. viscous damping, stiffness) matrix, $k$ being the number of DoF.

For $j \in \llbracket 1, q \rrbracket$, let $\mathcal{F}_{j}: u \mapsto \mathcal{F}_{j}(u)$, be $q$ linear forms which associate a scalar to a time function $u$. For a vector $\underline{u}=\left\{u_{1}, u_{2}, \ldots, u_{k}\right\}^{t}$, let note $\underline{\underline{F}}(\underline{u})$ the $k \times q$ matrix the $(i, j)$ coefficient of which is $\mathcal{F}_{j}\left(u_{i}\right)$ :

$$
\underline{\underline{\mathcal{F}}}(\underline{u})=\left(\begin{array}{cccc}
\mathcal{F}_{1}\left(u_{1}\right) & \mathcal{F}_{2}\left(u_{1}\right) & \ldots & \mathcal{F}_{q}\left(u_{1}\right) \\
\mathcal{F}_{1}\left(u_{2}\right) & \mathcal{F}_{2}\left(u_{2}\right) & \cdots & \mathcal{F}_{q}\left(u_{2}\right) \\
\vdots & \vdots & \ddots & \vdots \\
\mathcal{F}_{1}\left(u_{k}\right) & \mathcal{F}_{2}\left(u_{k}\right) & \cdots & \mathcal{F}_{q}\left(u_{k}\right)
\end{array}\right) .
$$

Applying the $q$ forms $\mathcal{F}_{j}$ to the Eq. (1), we obtain the algebraic equations system

$$
\underline{\underline{M}} \underline{\underline{\mathcal{F}}}(\underline{\ddot{x}})+\underline{\underline{C}} \underline{\underline{\mathcal{F}}}(\underline{\dot{x}})+\underline{\underline{K}} \underline{\underline{\mathcal{F}}}(\underline{x})=\underline{\underline{\mathcal{F}}}(\underline{f}) .
$$

Let us note the vector $\underline{X}$ built from the coefficients of matrices $\underline{\underline{M}}, \underline{\underline{K}}$ and $\underline{\underline{C}}$. Rearranging the terms of Eq. (2), the problem can be written in the form

$$
\underline{\underline{A}} \underline{X}=\underline{B},
$$

where $\underline{\underline{A}}$ is a rectangular matrix. When the square matrix $\underline{\underline{A}}^{t} \underline{\underline{A}}$ is invertible, inverting this system in the sense of least squares leads to the normal equation

$$
\underline{X}=\left(\underline{\underline{A}}^{t} \underline{\underline{A}}\right)^{-1} \underline{\underline{A}}^{t} \underline{B},
$$

and permits then to identify the coefficients of $\underline{\underline{M}}, \underline{\underline{K}}$ and $\underline{\underline{C}}$. Eq. (3) will be proposed

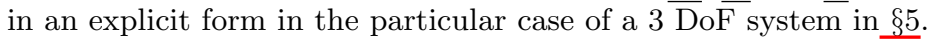

In the case of complex linear forms $\mathcal{F}_{j}$ (like CCWT, see $\underline{\underline{\underline{4}}}$ ), the matrices $\underline{\underline{A}}$ and $\underline{B}$ are replaced by $(\Re(\underline{\underline{A}}), \Im(\underline{\underline{A}}))^{t}$ and $(\Re(\underline{B}), \Im(\underline{B}))^{t}$ respectively where $\Re$ and $\widetilde{\overline{ }} \Im$ are 
the real and imaginary part respectively. The size of the system to invert depend to the hypothesis made on the matrix to identify. Assuming $\underline{\underline{M}}$ diagonal and $\underline{\underline{K}}$ and $\underline{\underline{C}}$ symetrical, the number of terms to identify is $\left(k^{2}+2 k\right)$. The size of the system is thus $q \times\left(k^{2}+2 k\right)\left(2 q \times\left(k^{2}+2 k\right)\right.$ in the case of complex linear forms $)$.

In the two following paragraphs, the $\mathcal{F}_{j}$ applications will be defined as projection on a polynomial basis and as continuous wavelet transform in order to compare both approaches in term of performance and robustness to noise.

\section{Identification procedure with Chebyshev polynomials}

3.1 Chebyshev polynomials

For $s \in \mathbb{N}$, let $T_{s}$ be the Chebyshev polynomials of the first kind

$$
T_{s}(\tau)=\cos (s \arccos \tau), \forall \tau \in[-1,1] .
$$

The set $\left\{T_{0}, T_{1}, \ldots, T_{p}\right\}$ forms an orthogonal basis for the set of all polynomials of degree lower or equal to $p$ with respect to the scalar product

$$
\langle f, g\rangle=\int_{-1}^{1} \frac{f(\tau) g(\tau)}{\sqrt{1-\tau^{2}}} d \tau .
$$

The derivative of each polynomial can be expressed as a sum of polynomials of lower order, that allows to write

$$
\frac{d \underline{T}}{d \tau}=\underline{\underline{D}} \underline{T}
$$

where $\underline{T}=\left(T_{0}, T_{1}, \ldots, T_{p}\right)^{t}$ and $d \underline{T} / d \tau=\left(d T_{0} / d \tau, d T_{1} / d \tau, \ldots, d T_{p} / d \tau\right)^{t}$. The expression of the matrix $\underline{\underline{D}}$ is given in the appendix A.1.

3.2 Expansion of a function on a Chebyshev basis

Let $u: t \mapsto u(t)$ be a function defined on the interval $\left[t_{\min }, t_{\max }\right]$ and let $\breve{u}: \tau \mapsto \check{u}(\tau)$ be the associated function defined for $\tau \in[-1,1]$ by

$$
\check{u}(\tau(t))=u(t),
$$

where $\tau(t)=\left(2 t-t_{\max }-t_{\min }\right) /\left(t_{\max }-t_{\min }\right)$. In this paragraph we are interested in the expansion $P_{p}[u]$ of $u$ on the basis $\left\{T_{0}, T_{1}, \ldots, T_{p}\right\}$

$$
P_{p}[u](t)=\sum_{s=0}^{p} \frac{\left\langle\check{u}, T_{s}\right\rangle}{\left\langle T_{s}, T_{s}\right\rangle} T_{s}(\tau(t))=\underline{\alpha} \underline{T}(\tau(t)),
$$

where the notation $\underline{\alpha}=\left(\alpha_{0}, \alpha_{1}, \ldots, \alpha_{p}\right)$, with $\alpha_{s}=\left\langle\check{u}, T_{s}\right\rangle /\left\langle T_{s}, T_{s}\right\rangle$ has been introduced. The values of $u$ are supposed to be known in a finite number of sampled instants. To estimate the coefficients of the expansion $P_{p}[u]$ on the Chebychev basis, it is assumed that the function $u$ is piecewise affine between these instants. The coefficients of the expansion of $u$ are then given by

$$
\left\langle\check{u}, T_{s}\right\rangle=\int_{-1}^{1} \frac{\check{u}(\tau) \cos (s \arccos \tau)}{\sqrt{1-\tau^{2}}} d \tau,
$$


which the calculation is given in the appendix A.2.

For example, the 1000 first coefficients of the projection of the sum of exponentially damped harmonic signals at $5 \mathrm{~Hz}, 10 \mathrm{~Hz}$ and $20 \mathrm{~Hz}$

$$
v(t)=0.8 e^{-2 t} \sin (10 \pi t)+0.4 e^{-t} \sin (20 \pi t)+0.5 e^{-3 t} \sin (40 \pi t),
$$

plotted on Fig. 1, discretized on a period of $4 \mathrm{~s}$ with a sample frequency of $1000 \mathrm{~Hz}$, are represented on Fig. 2.

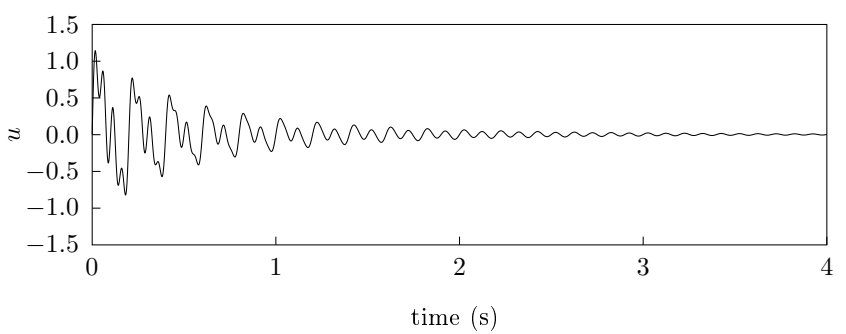

Fig. 1 Signal $v$, defined by Eq. (7)

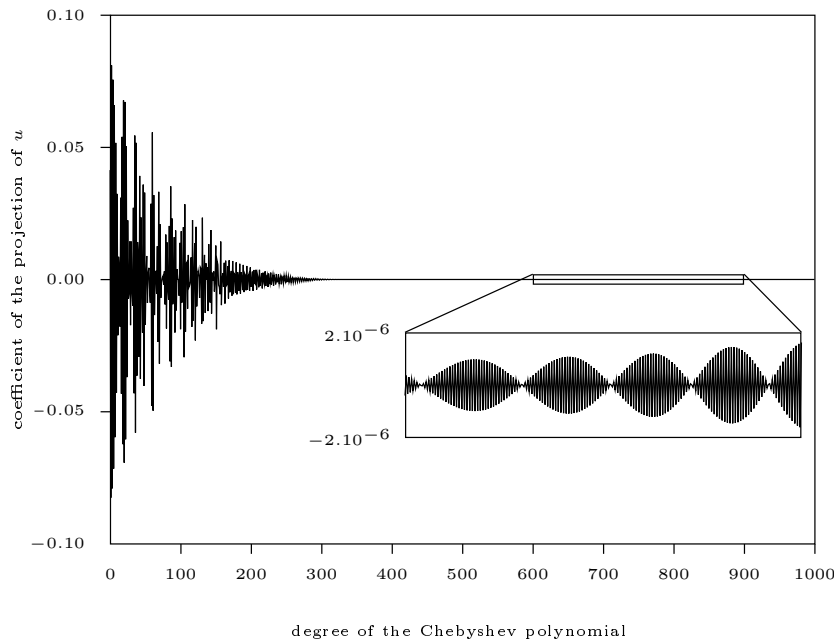

Fig. 2 Coefficients on the Chebyshev basis of signal $v$

Assuming that the expansion of the derivative of a signal $u$ on the basis is close to the derivative of the expansion of $u$, that is

$$
P_{p}[\dot{u}](t) \simeq \frac{d}{d t} P_{p}[u](t)=\frac{2}{t_{\max }-t_{\min }} \underline{\alpha} \underline{\underline{D}} \underline{T}(\tau(t)),
$$


the coefficients of the expansion of $\dot{u}$ can be calculated from those of the expansion of $u$. In the same way, we have

$$
P_{p}[\ddot{u}](t) \simeq\left(\frac{2}{t_{\max }-t_{\min }}\right)^{2} \underline{\alpha} \underline{D}^{2} \underline{T}(\tau(t)) .
$$

Note that to obtain the expansion of $\ddot{u}$ on polynomials of degrees 0 to $p$, the expansion of $u$ has to be calculated on polynomials of degrees 0 to $p+2$ (the degree of the derivative of a polynomial is the degree of the polynomial -1 , which explain the column of zeros in the matrix $\underline{\underline{D}}$ ).

For the signal of Fig. 1, analytically known, let observe the error then made on the coefficients of $P_{p}[\ddot{u}]$. The distance between the coefficients directly computed and those estimated with Eq. (9) is plotted versus the size $p$ of the basis on the Fig. 3. We

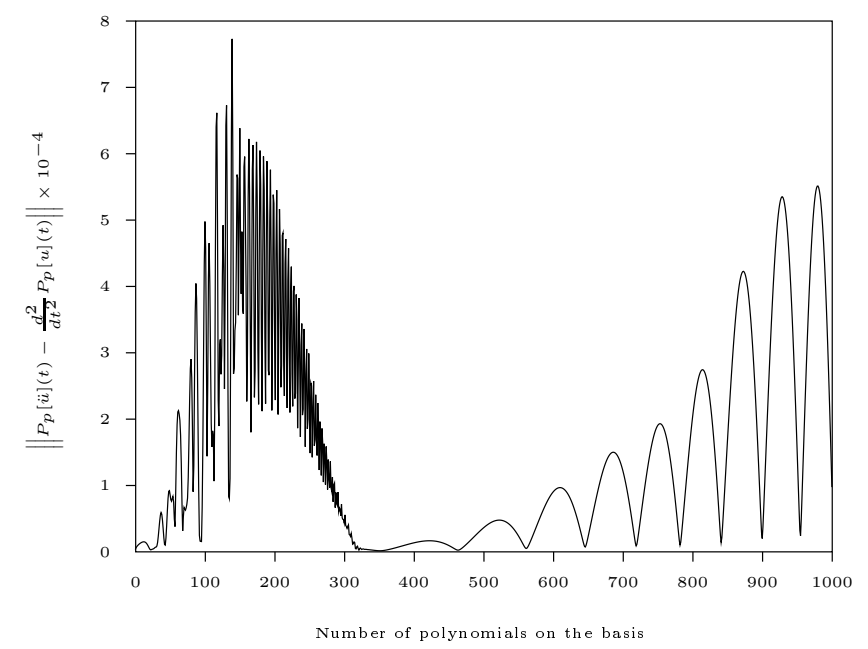

Fig. 3 Distance between $P_{p}[\ddot{u}](t)$ and $\frac{d^{2}}{d t^{2}} P_{p}[u](t)$, versus the size of the Chebychev basis

observe that this error is large when the signal is expanded on a number of polynomials insufficient to describe it correctly ( $p<300$ in our case). But when the signal is expanded on a wide basis, the coefficients of $P_{p}[\ddot{u}]$ are not correctly estimated using Eq. (9). This is due to small coefficients of the expansion of $u$ for polynomials of large degrees $s$ (see Fig. 2) which are amplified when multiply by the terms of matrix $D$. This coefficients are unuseful for the description of the signal $u$, and conducts-to bad estimation of its derivatives. We decide thus to choose the size $p$ of the basis as the smallest integer such that all coefficients for $s>p$ are smaller than a threshold, defined as the larger computed coefficient $\times 10^{-4}$.

In previous work (Rémond et al, 2008), the coefficients of the expansion of a signal was calculated in the sens of root mean square : for a given integer $p$, the signal was estimated by the sum of Chebychev polynomial of degrees $\leq p$ which is the nearest as possible to the signal at each instants $t_{i}$. This conducted to ill estimation of signals if the size of the basis was badly chosen, in particular in the case of a to-large basis. With the definition of the expansion used here, the larger is the basis, the better is 
the estimation of signals. Nevertheless, the size of the basis has to be limited because of the effect of large coefficients of the matrix $\underline{D}$ when the expansion is derived : the choice of the size of the basis is linked to the estimation of the derivatives of the signal, not to the estimation of the signal itself. On Fig. 4, it can be seen that the proposed criterion for this size leads to good estimation of acceleration in the major part of the time window, but it persists edge effect, where the estimation is not good.

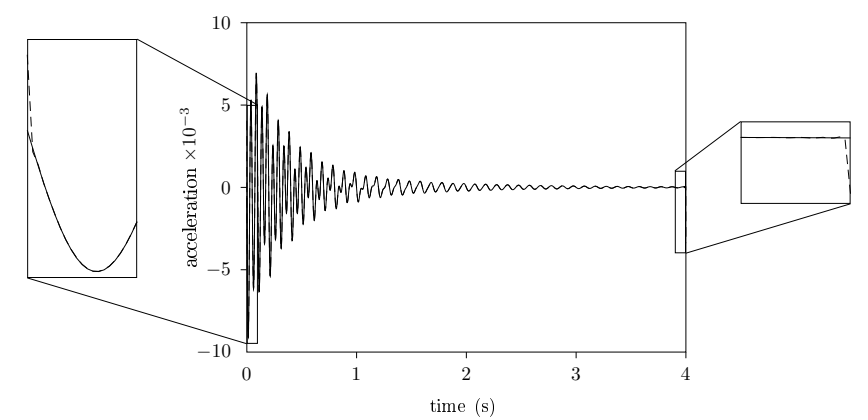

Fig. 4 Edge effect on the estimation of the acceleration

\subsection{Identification procedure}

Let us now see how expansion of signals under Chebyshev basis can be used to apply the identification method described in $\S 2$. Two definitions can be used for the linear forms applied to the equation of motion. Each application $\mathcal{F}_{j}$ can be defined as the coefficient of the expansion of $u$ on the $j^{\text {th }}$ Chebyshev polynomial

$$
\mathcal{F}_{j}(u)=\left\langle\check{u}, T_{j}\right\rangle /\left\langle T_{j}, T_{j}\right\rangle
$$

or as the projection of the signal $u$, computed at a given instant $t_{j}$

$$
\mathcal{F}_{j}(u)=P_{p}[u]\left(t_{j}\right)
$$

Note that defining $\mathcal{F}_{j}$ with Eq. (11) lead to invert a system made of linear combinations of the system obtain when defining $\mathcal{F}_{j}$ with Eq. (10), coefficients of these combinations being the values of the Chebyschev polynomials at $t_{j}$. Both definitions have been tested, and it has been observed that the second one conducts to better results of identification. Indeed, it has been shown in the previous paragraph that, at instants near the edges of the time window, signals, especially second derivatives, are not well estimated by their expansions. Using Eq. (11) for the identification permits to choose values of instants $t_{j}$ far from the edges, and thus reduce the error made on the results of identification.

The values of $\mathcal{F}_{j}(\dot{u})$ and $\mathcal{F}_{j}(\ddot{u})$ are deduced from those of $\mathcal{F}_{j}(u)$ by applying Eq. (8) and (9). 
4 Identification procedure with Cauchy wavelet analysis

4.1 Continuous Cauchy wavelet transform

The Cauchy Continuous Wavelet Transform (CCWT) of a real signal $u(t)$ of finite energy is defined by

$$
T_{\psi_{n}}[u](b, a)=\frac{1}{a} \int_{-\infty}^{+\infty} u(t) \bar{\psi}_{n}\left(\frac{t-b}{a}\right) d t,
$$

where the mother wavelet is chosen as the standard Cauchy wavelet with order $n$ :

$$
\psi_{n}(t)=\left(\frac{i}{t+i}\right)^{n+1},
$$

and $\bar{\psi}_{n}(\cdot)$ is its complex conjugate. The real parameters $a>0$ and $b$ introduce scaledilatation and time-translation, respectively. An alternative expression of the CCWT can be obtained by applying Parseval's theorem to Eq. (12)

$$
T_{\psi_{n}}[u](b, a)=\frac{1}{2 \pi} \int_{-\infty}^{+\infty} \hat{u}(\omega) \hat{\psi}_{n}(a \omega) e^{i \omega b} d \omega
$$

where $\hat{\psi}_{n}(\omega)$ is the Fourier transform of the mother wavelet :

$$
\hat{\psi}_{n}(\omega)=\int_{-\infty}^{+\infty} \psi_{n}(t) e^{-i \omega t} d t=\frac{2 \pi \omega^{n} e^{-\omega}}{n !} H(\omega),
$$

where $H(\cdot)$ is the Heaviside function.

The scale parameter $a$ plays the role of the inverse of frequency. To visualize the CCWT in the time-frequency plane rather than in the time-scale plane for more readability, we have thus to define a correspondence between the scale $a$ and the angular frequency $\xi$. We choose it so that the absolute value of the CCWT of an harmonic signal $w(t)=A \cos \left(\omega_{0} t\right)$, where $A$ is a positive constant, exhibits maxima for $\xi=\omega_{0}$, that is

$$
a=\frac{n}{\xi} .
$$

Indeed, the CCWT of $w$ is deduced from Eq. (13)

$$
T_{\psi_{n}}[w](b, a)=\frac{A}{2} \hat{\psi}_{n}\left(a \omega_{0}\right) e^{i \omega_{0} b}
$$

and the absolute value of the Fourier transform of the Cauchy wavelet $\left|\hat{\psi}_{n}(\cdot)\right|$ is peaked at the value of the angular frequency equal to $n$ (see Eq. (14)), and thus the absolute value of the CCWT of $w(t)$ is maximum when $a \omega_{0}=n$.

The local resolution of the CCWT in time and frequency can be estimated by introducing the duration $\Delta t$ and bandwidth $\Delta \omega$ of the translated and scaled mother wavelet $\psi_{n}((\cdot-b) / a)$. Defined in terms of root mean squares, they are given, for the Cauchy wavelet, by

$$
\Delta t=\frac{a}{\sqrt{2 n-1}}, \quad \Delta \omega=\frac{\sqrt{2 n+1}}{2 a},
$$

(Le and Argoul, 2004). The $Q$ factor can be also introduced to characterise the resolution of the mother wavelet and then of the CCWT. It is defined as the center-frequency 
and the frequency bandwidth of the mother wavelet, and is equal, for the Cauchy wavelet, to

$$
Q=\frac{1}{2} \sqrt{2 n+1} .
$$

The choice of the parameter $n$ of the Cauchy wavelet is thus important to optimize the parameter identification procedure, as it will be seen in the following paragraphs.

4.2 Adaptative continuous Cauchy wavelet transform

For a given Cauchy mother wavelet, i.e. a given parameter $n$, the resolution of the CCWT depends on the scale $a$, as we can see on Eq. (17). The resolution is thus not uniform in the time-frequency plane. It could be interesting to have an uniform resolution, and that is why an adaptative CCWT is defined hereafter.

Assuming $n>>1$, so that $2 n+1 \simeq 2 n-1 \simeq 2 n$, the duration $\Delta t$ and bandwidth $\Delta \omega$ of the translated and scaled mother wavelet are given by

$$
\Delta t \simeq \frac{1}{\xi} \sqrt{\frac{n}{2}}, \quad \Delta \omega \simeq \frac{\xi}{\sqrt{2 n}},
$$

and the product

$$
\Delta t \Delta \omega=\frac{1}{2} \sqrt{1+\frac{2}{2 n-1}} \simeq \frac{1}{2}
$$

is almost a constant independent of $n$. To have an uniform resolution in the timefrequency plane, we decide to make the order $n$ of the Cauchy wavelet dependent on the frequency $\xi$. So, $\Delta \omega$ is fixed and $n$ is defined as

$$
n_{\Delta \omega}(\xi)=\frac{1}{2}\left(\frac{\xi}{\Delta \omega}\right)^{2} .
$$

According to Eq (18), the resolution in frequency is thus the same everywhere in the time-frequency plane, and the product $\Delta t \Delta \omega$ being independent of $n$, so is the resolution in time.

\section{schema du plan temps-freq avec des rectangles}

The absolute value of the CCWT of the harmonic signal $w(t)=A \cos \left(\omega_{0} t\right)$, where $A$ is a positive constant, taken at $\xi=\omega_{0}$, is given by

$$
\left|T_{\psi_{n}}[w]\left(b, a=\frac{n}{\omega_{0}}\right)\right|=\frac{A}{2}\left|\hat{\psi}_{n}(n)\right|=A \frac{\pi n^{n} e^{-n}}{n !},
$$

(see Eq. (16)). Thus, we propose to normalize the value of the CCWT by $\pi n^{n} e^{-n} / n$ !, so that the values obtained at $\xi=\omega_{0}$ for an harmonic signal of angular frequency $\omega_{0}$ will correspond to the amplitude of the signal.

So, the adaptative CCWT of a real signal of finite energy $u(t)$ is defined, for a given bandwidth $\Delta \omega$, by

$$
S_{\Delta \omega}[u](b, \xi)=\frac{n_{\Delta \omega}(\xi) !}{\pi n_{\Delta \omega}(\xi)^{n \Delta \omega}(\xi) e^{-n_{\Delta \omega}(\xi)}} T_{\psi_{n_{\Delta \omega}(\xi)}}[u]\left(b, \frac{n_{\Delta \omega}(\xi)}{\xi}\right),
$$

where $b$ and $\xi$ represent the time and the angular frequency respectively, and $n(\xi)$ is defined by Eq (20). 
Examine now the edge effects. According to Erlicher and Argoul (2007), for a given $(b, a)$ time-scale point, the computation of $T_{\psi_{n}}[u](b, a)$ depends mainly on the signal values $u(t)$ occuring for $t$ belonging to the interval $\left[b-c_{t} \Delta t, b+c_{t} \Delta t\right]$ where $c_{t} \simeq 5$. The points $(b, a)$ where edge effects are negligible, verify therefore

$$
t_{\min }+c_{t} \Delta t \leq b \leq t_{\max }-c_{t} \Delta t .
$$

Introducing Eq. (19) in the latest inequality, we obtain

$$
t_{\min }+\frac{c_{t}}{2 \Delta \omega} \leq b \leq t_{\max }-\frac{c_{t}}{2 \Delta \omega} .
$$

In the case of a modulated amplitude and phase signal

$$
w(t)=\sum_{i=1}^{k} A_{i}(t) \cos \left(\alpha_{i}(t)\right)
$$

where each amplitude $A_{i}(t)$ varies slowly compared to the corresponding phase $\alpha_{i}(t)$, the energy of the CCWT of the signal tends to localize around a set of points in the time-scale plane called ridges which are defined as

$$
R_{i}[w]=\left\{(b, \xi) \mid \xi=\dot{\alpha}_{i}(b)\right\} \quad \forall i \in \llbracket 1, k \rrbracket .
$$

As an example, the absolute value of the adaptative CCWT of the signal $v$ defined by Eq. (7) is represented on Fig. 5, with different values of $\Delta \omega$. We can see that there is a compromise to do on the choice of $\Delta \omega$ : when its value is small, the characteristic frequencies of the signal are clearly separated, while they are not with a large value, but edge effect may become important in this case.

\subsection{Identification procedure}

For a given bandwidth $\Delta \omega$ and a given time-frequency point $\left(b_{j}, \xi_{j}\right)$, the application $\mathcal{F}_{j}$ is defined as the adaptative CCWT, computed at $\left(b_{j}, \xi_{j}\right)$ :

$$
\mathcal{F}_{j}(u)=S_{\Delta \omega}[u]\left(b_{j}, \xi_{j}\right) .
$$

The CCWT of the derivative and the second derivative of a signal can be derived from the one of the signal by using mother wavelets $\dot{\psi}_{n}$ and $\ddot{\psi}_{n}$

$$
\begin{aligned}
& T_{\psi_{n}}[\dot{u}](b, a)=-\frac{1}{a} T_{\dot{\psi}_{n}}[u](b, a)=\frac{i(n+1)}{a} T_{\psi_{n+1}}[u](b, a), \\
& T_{\psi_{n}}[\ddot{u}](b, a)=\frac{1}{a^{2}} T_{\ddot{\psi}_{n}}[u](b, a)=-\frac{(n+1)(n+2)}{a^{2}} T_{\psi_{n+2}}[u](b, a) .
\end{aligned}
$$

The values of $\mathcal{F}_{j}(\dot{u})$ and $\mathcal{F}_{j}(\ddot{u})$ are then given by

$$
\begin{aligned}
& \mathcal{F}_{j}(\dot{u})=\frac{n_{j} !}{\pi n_{j} n_{j} e^{-n_{j}}} \frac{i\left(n_{j}+1\right) \xi_{j}}{n_{j}} T_{\psi_{n_{j}+1}}[u]\left(b_{j}, \frac{n_{j}}{\xi_{j}}\right), \\
& \mathcal{F}_{j}(\ddot{u})=-\frac{n_{j} !}{\pi n_{j} n_{j} e^{-n_{j}}} \frac{\left(n_{j}+1\right)\left(n_{j}+2\right) \xi_{j}^{2}}{n_{j}^{2}} T_{\psi_{n_{j}+2}}[u]\left(b, \frac{n_{j}}{\xi_{j}}\right),
\end{aligned}
$$

where the notation $n_{j}=n_{\Delta \omega}\left(\xi_{j}\right)$ has been introduced.

In order to derive benefit from the information present within the CCWT of the signals, the points $\left(b_{j}, \xi_{j}\right)$ of the time-frequency plane have to be chosen on the ridges. 


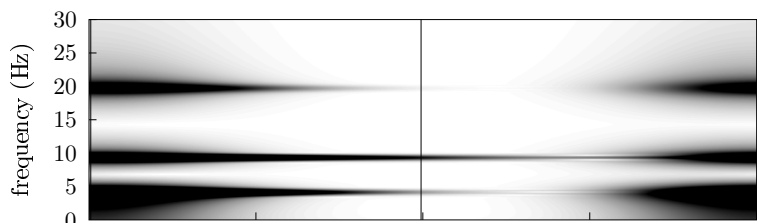

(a)

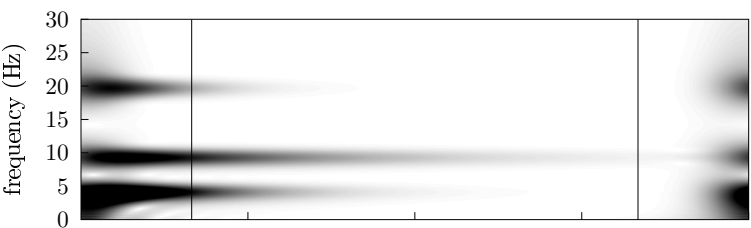

(b)

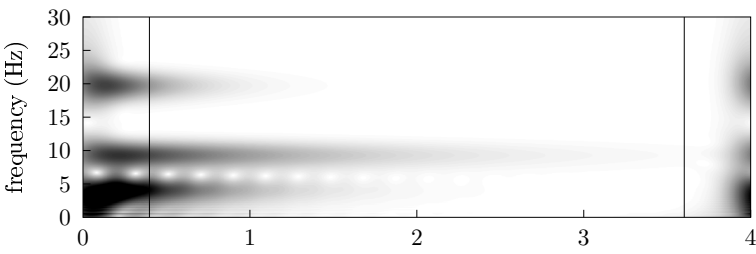

(c)

Fig. 5 Absolute value of adaptative CCWT of $v$ with (a) $\Delta \omega / 2 \pi=0.2 \mathrm{~Hz}$, (b) $\Delta \omega / 2 \pi=$ $0.6 \mathrm{~Hz}$, and (c) $\Delta \omega / 2 \pi=1 \mathrm{~Hz}$

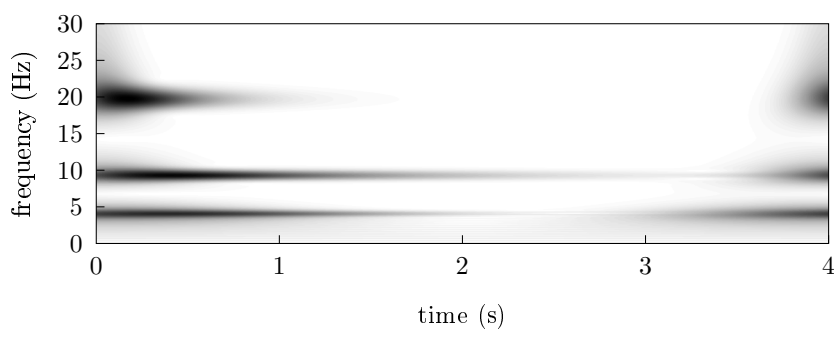

Fig. 6 Absolute value of CCWT of $v$ with $n=500$

\section{Validation by numerical simulations}

5.1 The case of a three degrees of freedom system

To illustrate the proposed identification method, the case of a 3 DoF of freedom linear system where $\underline{M}$ is diagonal and $\underline{C}$ and $\underline{K}$ are symetrical is considered. These matrices can be written as

$$
\underline{\underline{M}}=\left(\begin{array}{ccc}
m_{11} & 0 & 0 \\
0 & m_{22} & 0 \\
0 & 0 & m_{33}
\end{array}\right), \quad \underline{\underline{C}}=\left(\begin{array}{ccc}
c_{11} & c_{12} & c_{13} \\
c_{12} & c_{22} & c_{23} \\
c_{13} & c_{23} & c_{33}
\end{array}\right), \quad \underline{\underline{K}}\left(\begin{array}{lll}
k_{11} & k_{12} & k_{13} \\
k_{12} & k_{22} & k_{23} \\
k_{13} & k_{23} & k_{33}
\end{array}\right),
$$

and the vector of the 15 coefficients to be identified can be expressed as

$$
\underline{X}=\left(m_{11}, m_{22}, m_{33}, c_{11}, c_{12}, c_{13}, c_{22}, c_{23}, c_{33}, k_{11}, k_{12}, k_{13}, k_{22}, k_{23}, k_{33}\right)^{t} .
$$


In this specific case, the matrix $\underline{\underline{A}}=\left(\underline{\underline{A}}_{1}, \underline{\underline{A}}_{2}, \underline{\underline{A}}_{3}\right)$ and $\underline{B}$ of the Eq. (3) are then given by

$$
\begin{aligned}
& \underline{\underline{A}}_{1}=\left(\begin{array}{ccc}
\underline{\mathcal{F}}\left(\ddot{x}_{1}\right)^{t} & \underline{0} & \underline{0} \\
\underline{0} & \underline{\mathcal{F}}\left(\ddot{x}_{2}\right)^{t} & \underline{0} \\
\underline{0} & \underline{0} & \underline{\mathcal{F}}\left(\ddot{x}_{3}\right)^{t}
\end{array}\right)
\end{aligned}
$$

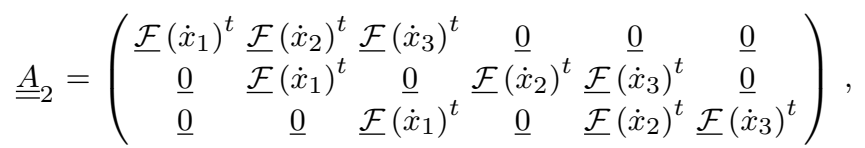

$$
\begin{aligned}
& \underline{\underline{A}}_{3}=\left(\begin{array}{cccccc}
\underline{\mathcal{F}}\left(x_{1}\right)^{t} & \underline{\mathcal{F}}\left(x_{2}\right)^{t} & \underline{\mathcal{F}}\left(x_{3}\right)^{t} & \underline{0} & \underline{0} & \underline{0} \\
\underline{0} & \underline{\mathcal{F}}\left(x_{1}\right)^{t} & \underline{0} & \underline{\mathcal{F}}\left(x_{2}\right)^{t} & \underline{\mathcal{F}}\left(x_{3}\right)^{t} & \underline{0} \\
\underline{0} & \underline{0} & \underline{\mathcal{F}}\left(x_{1}\right)^{t} & \underline{0} & \underline{\mathcal{F}}\left(x_{2}\right)^{t} & \underline{\mathcal{F}}\left(x_{3}\right)^{t}
\end{array}\right), \\
& \underline{B}=\left(\begin{array}{l}
\frac{\mathcal{F}}{\mathcal{F}}\left(f_{1}\right)^{t} \\
\underline{\mathcal{F}}\left(f_{2}\right)^{t} \\
\underline{\mathcal{F}}\left(f_{3}\right)^{t}
\end{array}\right)
\end{aligned}
$$

where the notation $\underline{\mathcal{F}}(u)=\left(\mathcal{F}_{1}(u), \mathcal{F}_{2}(u), \ldots, \mathcal{F}_{q}(u)\right)$ has been introduced.

\subsection{Definition of the system}

Two different systems are used for the numerical simulations. They have both the same architecture, illustrated on Fig. 7, and the same values of mass and stiffness, but the damping are modify in order to test the method on systems with more or less proportional damping. The mechanical parameters are collected in Tab. 1.

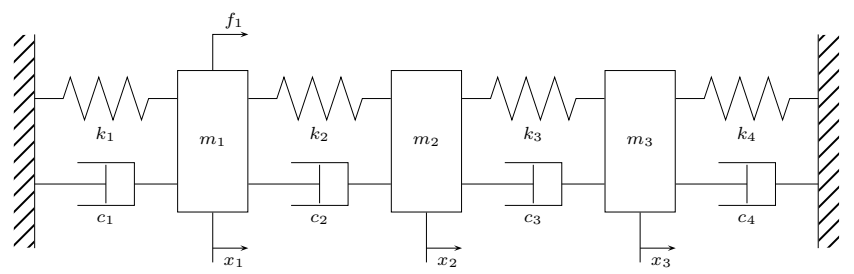

Fig. 7 Architecture of the 3 DoF systems used for numerical simulations

\begin{tabular}{lcccccc}
\hline \multicolumn{2}{l}{$\begin{array}{l}\text { Systems 1 and 2 } \\
\text { Mass }\end{array}$} & \multicolumn{2}{c}{ Systems 1 and 2 } & & System 1 & System 2 \\
Stiffness & $\mathrm{N} / \mathrm{m}$ & Damping & $\mathrm{Ns} / \mathrm{m}$ & $\mathrm{Ns} / \mathrm{m}$ \\
\hline$m_{1}$ & 1 & $k_{1}$ & 1000 & $c_{1}$ & 5 & 0.1 \\
$m_{2}$ & 2 & $k_{2}$ & 4000 & $c_{2}$ & 4 & 0.2 \\
$m_{3}$ & 1 & $k_{3}$ & 3000 & $c_{3}$ & 3 & 2 \\
& & $k_{4}$ & 5000 & $c_{4}$ & 2 & 20 \\
\hline
\end{tabular}

Table 1 Parameter values of the 3 DoF systems used for simulations 
The coefficients of the matrices $\underline{\underline{M}}, \underline{\underline{C}}$, and $\underline{\underline{K}}$ representing these systems are given in the Tab. 4 and 5. The modal properties (natural frequencies, damping ratios and modes shapes) of these systems are given in the Tab. 2 and 3. The index of the non-

\begin{tabular}{|c|c|c|c|c|}
\hline Mode & $\begin{array}{c}\text { Eigen frequency } \\
(\mathrm{Hz})\end{array}$ & $\begin{array}{c}\text { Damping ratio } \\
(\%)\end{array}$ & Shape & $\begin{array}{l}\text { Index of non } \\
\text { proportionality }\end{array}$ \\
\hline 1 & 4.8018 & 3.25 & $\left(\begin{array}{c}1 \\
1.0224+0.0223 i \\
0.4323+0.0169 i\end{array}\right)$ & 0.0130 \\
\hline 2 & 12.7390 & 5.02 & $\begin{array}{c}1 \\
-0.3502+0.0475 i \\
-0.6496-0.0619 i\end{array}$ & 0.0609 \\
\hline 3 & 15.2438 & 3.92 & $\begin{array}{c}1 \\
-1.0358+0.1353 i \\
2.5640-0.6208 i\end{array}$ & 0.0779 \\
\hline
\end{tabular}

Table 2 Modal characteristics of system 1

\begin{tabular}{ccccc}
\hline Mode & $\begin{array}{c}\text { Eigen frequency } \\
(\mathrm{Hz})\end{array}$ & $\begin{array}{c}\text { Damping ratio } \\
(\%)\end{array}$ & \multicolumn{1}{c}{ Shape } & $\begin{array}{c}\text { Index of non } \\
\text { proportionality }\end{array}$ \\
\hline 1 & 4.8051 & 2.29 & $\left(\begin{array}{c}1 \\
1.0223-0.0097 i \\
0.4305-0.0333 i\end{array}\right)$ & 0.0205 \\
1 & & & $\left(\begin{array}{r}1 \\
-0.3905-0.0923 i \\
-0.5401+0.2936 i \\
1\end{array}\right)$ & 0.2430 \\
3 & 12.9021 & 3.04 & $\left(\begin{array}{r}-0.9492-0.3906 i \\
1.7756+2.2255 i\end{array}\right)$ & 0.2941 \\
\hline
\end{tabular}

Table 3 Modal characteristics of system 2

proportionality of the $j^{\text {th }}$ complex mode $\phi_{j}$, belonging to the interval $[0,1]$, is defined (Adhikari, 2004) by

$$
I_{j}=\sqrt{1-\frac{\left|\varphi_{j} . \phi_{j}\right|^{2}}{\left\|\varphi_{j}\right\|^{2}\left\|\phi_{j}\right\|^{2}}}
$$

where $\varphi_{j}$ is the corresponding real normal mode of the associated undamped system.

\subsection{Results of the identification}

Three case of excitation are studied, the force being always applied on the mass 1 . The two first cases are pure harmonic excitations of $20 \mathrm{~Hz}$ and $14 \mathrm{~Hz}$ respectively. This frequencies are chosen to be located respectively close and far from the natural frequencies of the studied systems. The third case is a white noise excitation.

The responses in displacements of the system 1 to these excitations are represented on the Fig. 8, 11 and 14. Simulated signals are recorded during $4 \mathrm{~s}$ at a sampling 
frequency of $1000 \mathrm{~Hz}$. The coefficients of the expansions of these signals on the Chebychev polynomials basis are represented on the Fig. 9, 12 and 15, and their adaptative CCWT, computed with $\Delta \omega / 2 \pi=0.3 \mathrm{~Hz}$, are shown on the Fig. 10, 13 and 16.
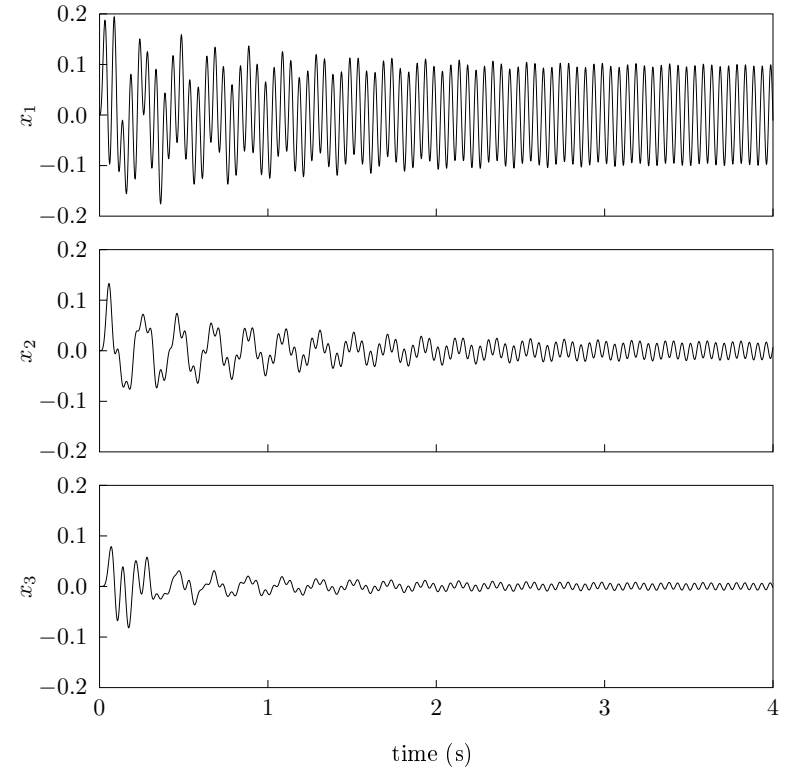

(a)

(b)

(c)

Fig. 8 Response of system 1 when mass 1 exciting by a pure harmonic force at $20 \mathrm{~Hz}$ (a) displacement of mass 1 (b) displacement of mass 2 (c) displacement of mass 3

Results of the identification of the system 1 (resp. system 2) with both methods are presented in Tab. 4 (resp. Tab. 5). For the method using Chebyshev basis, the

\begin{tabular}{lrrrrrrr}
\hline & Exact & \multicolumn{2}{c}{$20 \mathrm{~Hz}$} & \multicolumn{3}{c}{$14 \mathrm{~Hz}$} & \multicolumn{2}{c}{ BB } \\
& & $\mathrm{Ch}$. & $\mathrm{W}$. & $\mathrm{Ch}$. & $\mathrm{W}$. & $\mathrm{Ch}$. & $\mathrm{W}$. \\
\hline$m_{11}$ & 1 & 0.999986 & 1.000628 & 0.999997 & 1.001621 & 1.019296 & 1.000453 \\
$m_{22}$ & 2 & 1.999917 & 2.003621 & 1.999987 & 2.005292 & 2.031357 & 2.001023 \\
$m_{33}$ & 1 & 0.999951 & 1.001586 & 0.999988 & 1.001569 & 1.018734 & 1.001063 \\
\hline$c_{11}$ & 9 & 9.000056 & 8.998741 & 9.000077 & 9.003639 & 8.923262 & 8.997397 \\
$c_{12}$ & -4 & -3.999902 & -4.007103 & -4.000015 & -4.020535 & -3.958718 & -4.002256 \\
$c_{13}$ & 0 & -0.000001 & -0.000600 & -0.000017 & 0.022314 & -0.072171 & -0.045979 \\
$c_{22}$ & 7 & 6.999477 & 7.017934 & 7.000059 & 7.032804 & 7.027887 & 6.965176 \\
$c_{23}$ & -3 & -2.999798 & -3.007923 & -3.000070 & -3.018458 & -2.978610 & -2.919912 \\
$c_{33}$ & 5 & 4.999725 & 5.015449 & 5.000046 & 5.040925 & 5.015940 & 4.907940 \\
\hline$k_{11}$ & 5000 & 4999.81 & 5008.71 & 4999.98 & 5012.97 & 5083.74 & 5001.91 \\
$k_{12}$ & -4000 & -3999.83 & -4007.40 & -3999.98 & -4010.54 & -4065.28 & -4001.23 \\
$k_{13}$ & 0 & -0.00 & 0.14 & 0.01 & 1.26 & -2.47 & -1.66 \\
$k_{22}$ & 7000 & 6999.70 & 7012.20 & 6999.95 & 7018.09 & 7115.27 & 7001.98 \\
$k_{23}$ & -3000 & -2999.86 & -3004.45 & -2999.98 & -3007.50 & -3054.01 & -2999.52 \\
$k_{33}$ & 8000 & 7999.63 & 8011.73 & 7999.90 & 8013.35 & 8149.78 & 8003.71 \\
\hline
\end{tabular}

Table 4 Results of the identification of the system 1 with both methods 


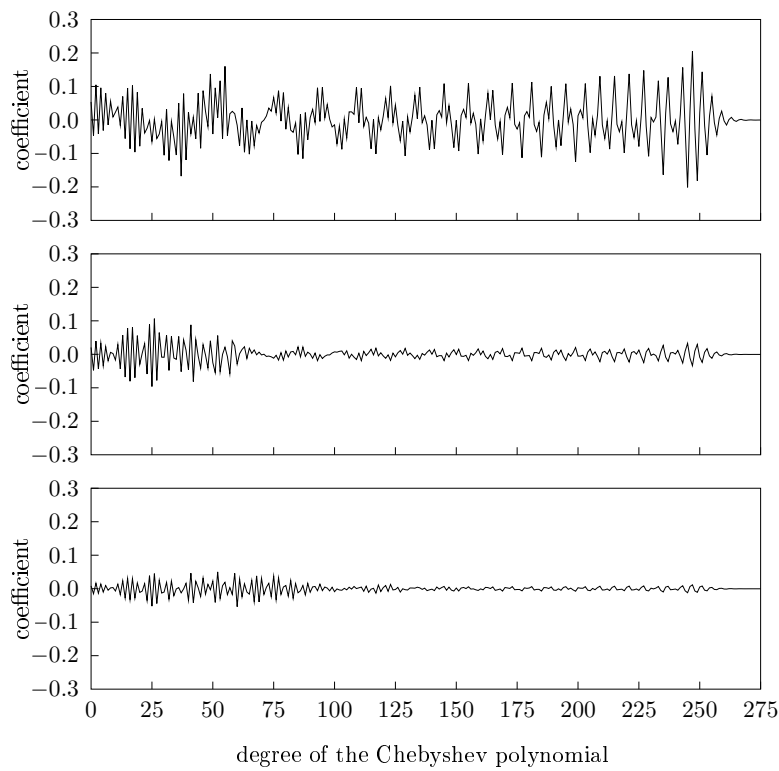

(a)

(b)

(c)

Fig. 9 Coefficients of the expansions of the displacements on the Chebychev basis when mass 1 exciting by a pure harmonic force at $20 \mathrm{~Hz}$ (a) expansion of $x_{1}$ (b) expansion of $x_{2}$ (c) expansion of $x_{3}$

\begin{tabular}{lrrrrrrr}
\hline & Exact & \multicolumn{2}{c}{$20 \mathrm{~Hz}$} & \multicolumn{2}{c}{$14 \mathrm{~Hz}$} & \multicolumn{2}{c}{ BB } \\
& & Ch. & W. & Ch. & W. & Ch. & W. \\
\hline$m_{11}$ & 1 & 0.999992 & 1.000172 & 0.999982 & 0.998917 & 1.018417 & 1.007453 \\
$m_{22}$ & 2 & 1.999954 & 2.000890 & 1.999925 & 1.997579 & 2.034522 & 2.012270 \\
$m_{33}$ & 1 & 0.999972 & 0.999597 & 0.999977 & 0.996854 & 1.018806 & 1.014040 \\
\hline$c_{11}$ & 0.3 & 0.299985 & 0.300452 & 0.300538 & 0.302464 & 0.176170 & 0.254454 \\
$c_{12}$ & -0.2 & -0.200053 & -0.200980 & -0.199369 & -0.218914 & -0.123997 & -0.152525 \\
$c_{13}$ & 0 & -0.000014 & 0.000029 & -0.000150 & 0.000946 & -0.073870 & 0.011959 \\
$c_{22}$ & 2.2 & 2.199751 & 2.204207 & 2.200905 & 2.224686 & 2.146662 & 2.154692 \\
$c_{23}$ & -2 & -1.999905 & -1.998600 & -2.000056 & -1.990790 & -1.995649 & -2.020780 \\
$c_{33}$ & 22 & 21.999395 & 21.990246 & 21.999206 & 21.947288 & 22.471849 & 22.247741 \\
\hline$k_{11}$ & 5000 & 4999.89 & 5002.39 & 4999.88 & 4993.87 & 5086.55 & 5035.14 \\
$k_{12}$ & -4000 & -3999.91 & -4002.04 & -3999.87 & -3994.55 & -4071.49 & -4028.16 \\
$k_{13}$ & 0 & -0.00 & -0.02 & -0.01 & 0.49 & 5.10 & -2.98 \\
$k_{22}$ & 7000 & 6999.83 & 7002.27 & 6999.78 & 6988.57 & 7131.15 & 7053.83 \\
$k_{23}$ & -3000 & -2999.92 & -2998.85 & -2999.94 & -2991.60 & -3070.52 & -3035.55 \\
$k_{33}$ & 8000 & 7999.79 & 7996.99 & 7999.82 & 7975.47 & 8171.89 & 8103.90 \\
\hline
\end{tabular}

Table 5 Results of the identification of the system 2 with both methods

expansions of the signals are considered at all the instants where original signals are sampled (of the number of 4001), except the ten first instants and the ten last instants, in order to free from edge effects. For the method using CCWT, in each case of excitation, the ridges of the time-frequency plane are detected with the crazy climbers algorithm, and five points $\left(b_{j}, \xi_{j}\right)$ per ridge is considered for the identification. These points are shown on the Fig. 10, 13 and 16. 


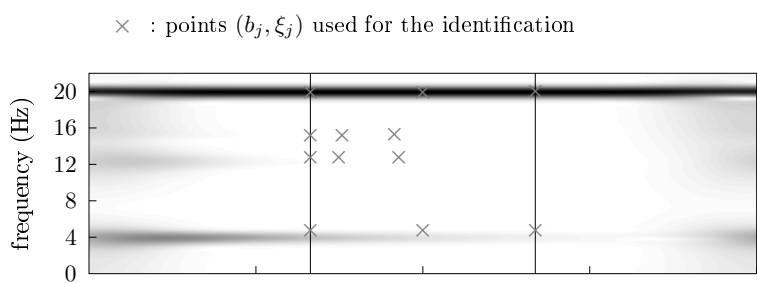

(a)

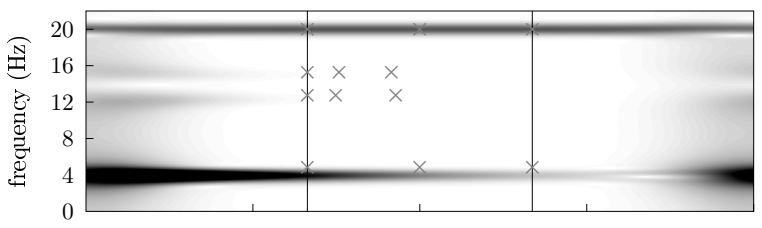

(b)

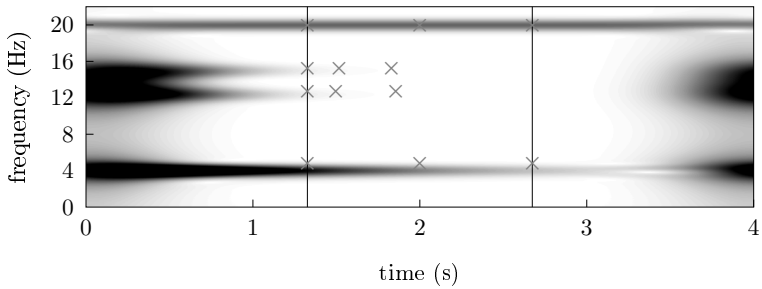

(c)

Fig. 10 Absolute value of adaptative CCWT of the displacements when mass 1 exciting by a pure harmonic force at $20 \mathrm{~Hz}$, computed with $\Delta \omega / 2 \pi=0.3 \mathrm{~Hz}$ (a) adaptative CCWT of $x_{1}$ (b) adaptative CCWT of $x_{2}$ (c) adaptative CCWT of $x_{3}$

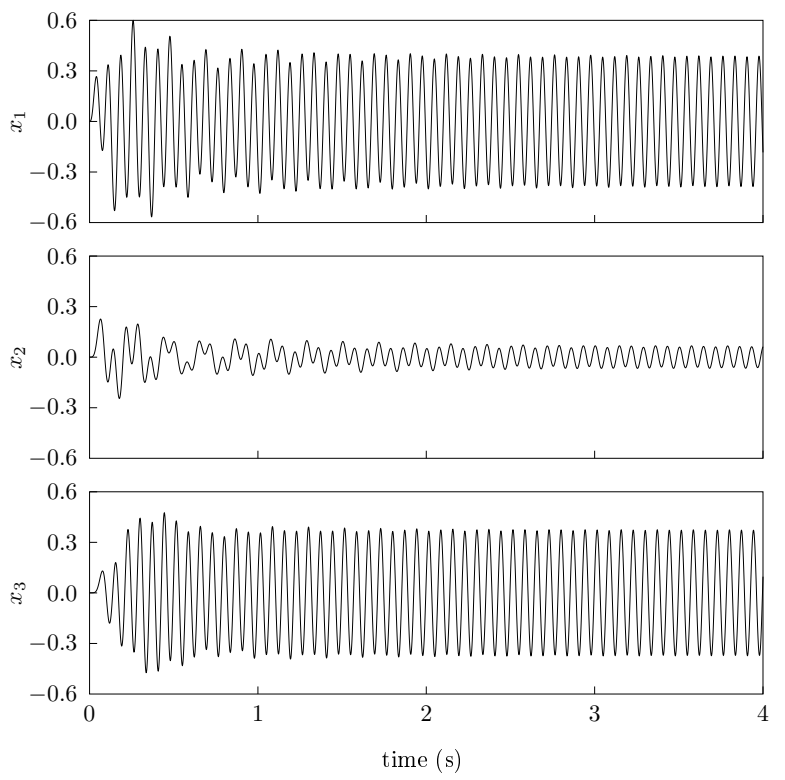

(a)

(b)

(c)

Fig. 11 Response of system 1 when mass 1 exciting by a pure harmonic force at $14 \mathrm{~Hz}$ (a) displacement of mass 1 (b) displacement of mass 2 (c) displacement of mass 3 


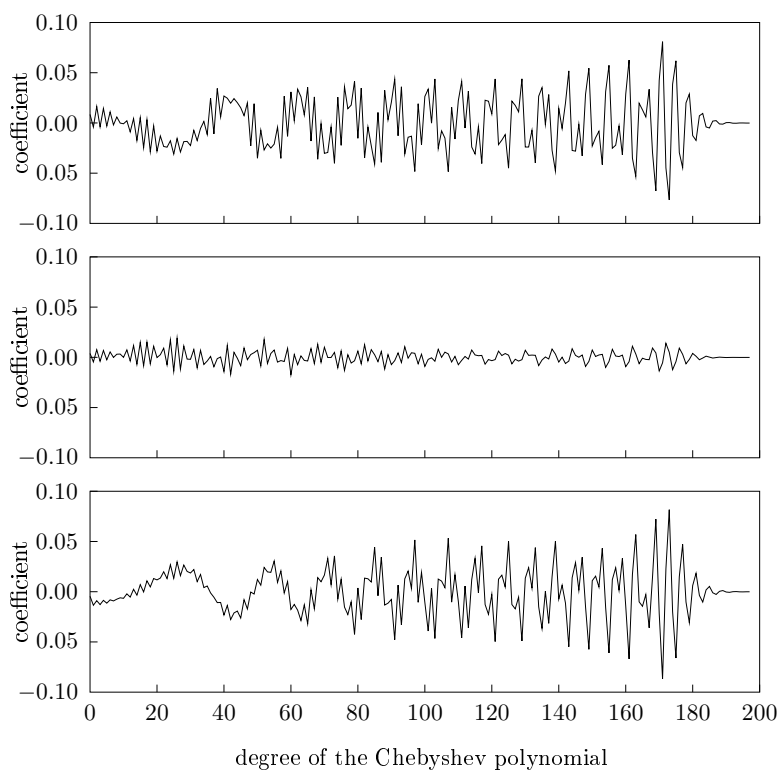

(a)

(b)

(c)

Fig. 12 Coefficients of the expansions of the displacements on the Chebychev basis when mass 1 exciting by a pure harmonic force at $14 \mathrm{~Hz}$ (a) expansion of $x_{1}$ (b) expansion of $x_{2}$ (c) expansion of $x_{3}$

Both methods give rather good results...

\subsection{Noise effect}

Noise is then added to the simulated signals. It is modeled by the centered normal distribution of variance $V_{b}$. To compare the level of the signal to the level of noise, we use the signal-to-noise ratio SNR, expressed in terms of the logarithmic decibel scale, and defined as

$$
\mathrm{SNR}=10 \log _{10} \frac{V_{s}}{V_{b}},
$$

where $V_{s}$ (resp. $V_{b}$ ) is the variance of the signal (resp. the noise).

Erreur identification definie par (sur Fig. 17)

$$
\begin{gathered}
3 \frac{\left(m_{11}-\tilde{m}_{11}\right)^{2}+\left(m_{22}-\tilde{m}_{22}\right)^{2}+\left(m_{33}-\tilde{m}_{33}\right)^{2}}{\left(\left|m_{11}\right|+\left|m_{22}\right|+\left|m_{33}\right|\right)^{2}} \\
+6 \frac{\left(c_{11}-\tilde{c}_{11}\right)^{2}+\left(c_{12}-\tilde{c}_{12}\right)^{2}+\left(c_{13}-\tilde{c}_{13}\right)^{2}+\left(c_{22}-\tilde{c}_{22}\right)^{2}+\left(c_{23}-\tilde{c}_{23}\right)^{2}+\left(c_{33}-\tilde{c}_{33}\right)^{2}}{\left(\left|c_{11}\right|+\left|c_{12}\right|+\left|c_{13}\right|+\left|c_{22}\right|+\left|c_{23}\right|+\left|c_{33}\right|\right)^{2}} \\
+6 \frac{\left(k_{11}-\tilde{k}_{11}\right)^{2}+\left(k_{12}-\tilde{k}_{12}\right)^{2}+\left(k_{13}-\tilde{k}_{13}\right)^{2}+\left(k_{22}-\tilde{k}_{22}\right)^{2}+\left(k_{23}-\tilde{k}_{23}\right)^{2}+\left(k_{33}-\tilde{k}_{33}\right)^{2}}{\left(\left|k_{11}\right|+\left|k_{12}\right|+\left|k_{13}\right|+\left|k_{22}\right|+\left|k_{23}\right|+\left|k_{33}\right|\right)^{2}}
\end{gathered}
$$


$\times$ : points $\left(b_{j}, \xi_{j}\right)$ used for the identification

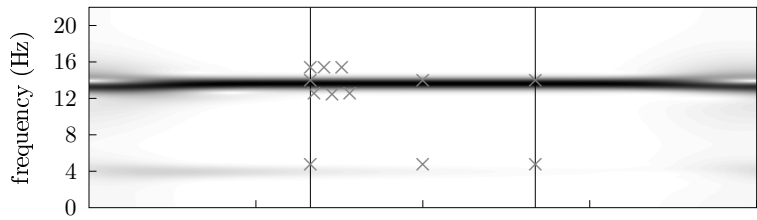

(a)

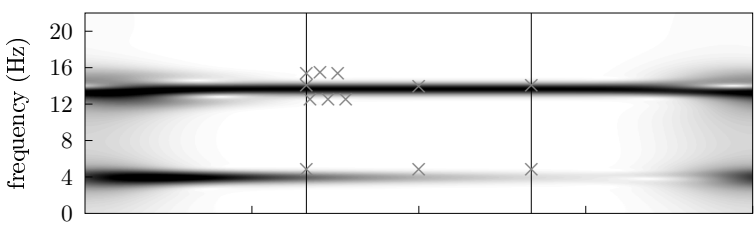

(b)

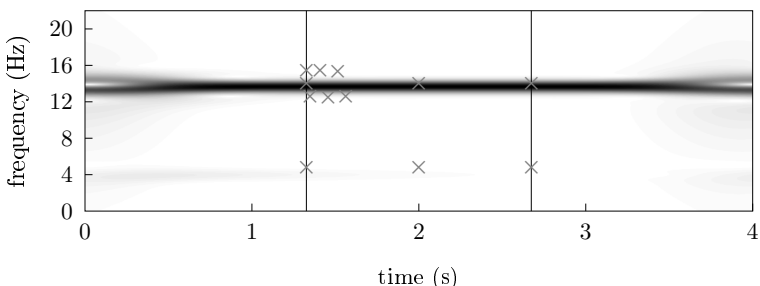

(c)

Fig. 13 Absolute value of adaptative CCWT of the displacements when mass 1 exciting by a pure harmonic force at $14 \mathrm{~Hz}$, computed with $\Delta \omega / 2 \pi=0.3 \mathrm{~Hz}$ (a) adaptative CCWT of $x_{1}$ (b) adaptative CCWT of $x_{2}$ (c) adaptative CCWT of $x_{3}$

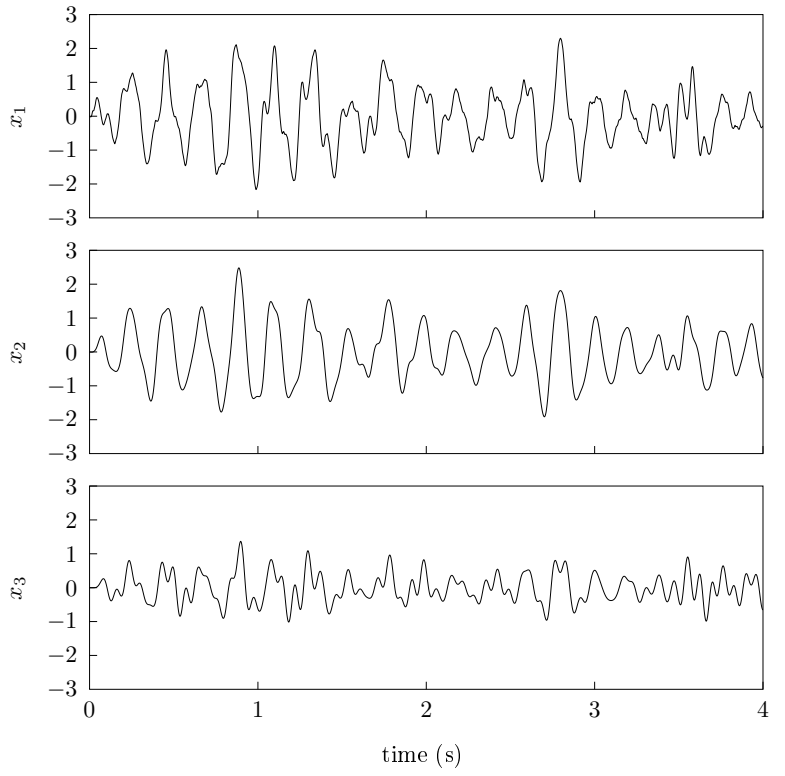

(a)

(b)

(c)

Fig. 14 Response of system 1 when mass 1 exciting by a white noise (a) displacement of mass 1 (b) displacement of mass 2 (c) displacement of mass 3 


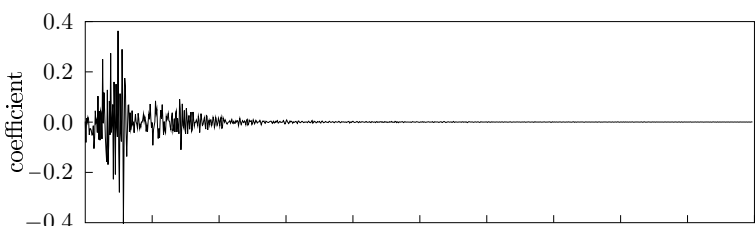

(a)

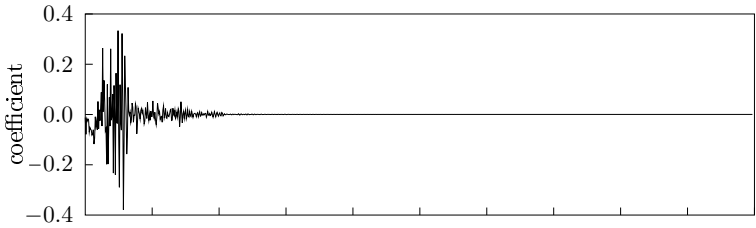

(b)

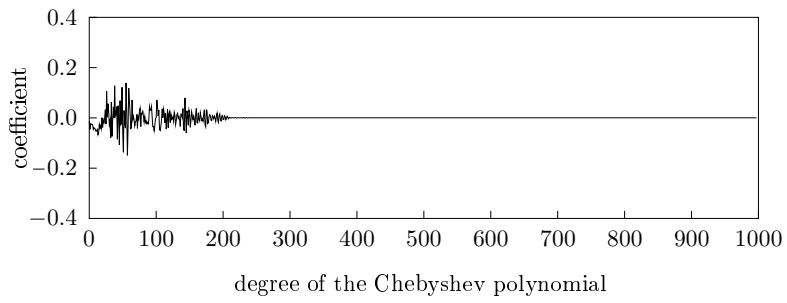

(c)

Fig. 15 Coefficients of the expansions of the displacements on the Chebychev basis when mass 1 exciting by a white noise (a) expansion of $x_{1}$ (b) expansion of $x_{2}$ (c) expansion of $x_{3}$ $\times$ : points $\left(b_{j}, \xi_{j}\right)$ used for the identification

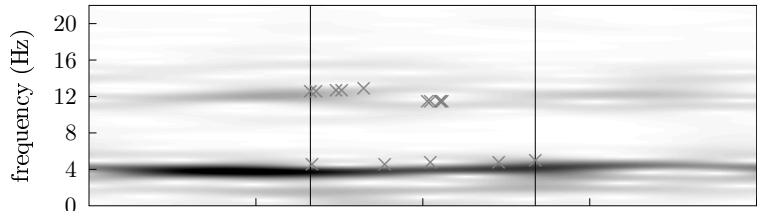

(a)

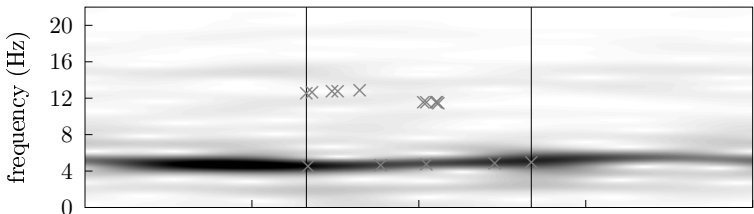

(b)

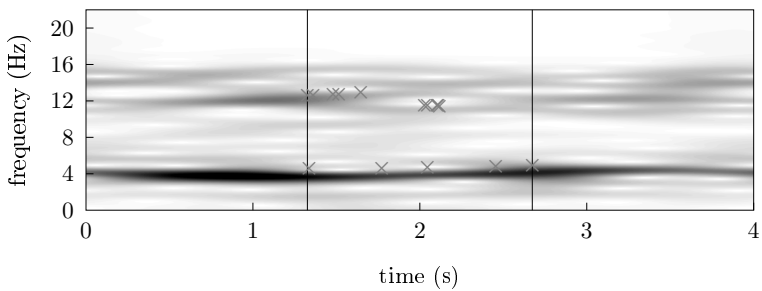

(c)

Fig. 16 Absolute value of adaptative CCWT of the displacements when mass 1 exciting by a white noise, computed with $\Delta \omega / 2 \pi=0.3 \mathrm{~Hz}$ (a) adaptative CCWT of $x_{1}$ (b) adaptative CCWT of $x_{2}$ (c) adaptative CCWT of $x_{3}$ 


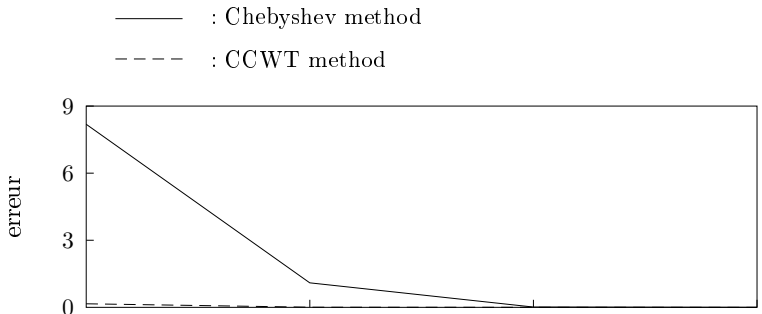

(a)

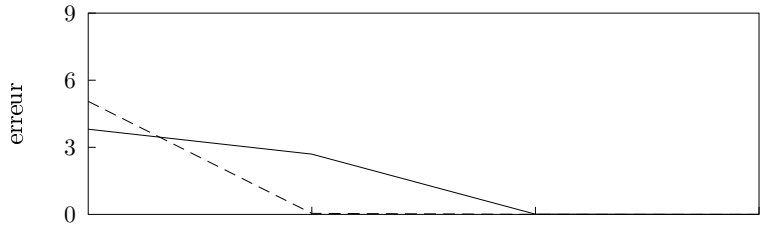

(b)

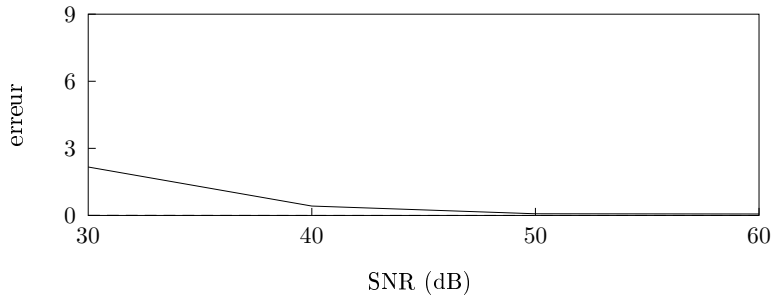

(c)

Fig. 17 Absolute value of adaptative CCWT of the displacements when mass 1 exciting by a white noise, computed with $\Delta \omega / 2 \pi=0.3 \mathrm{~Hz}$ (a) $20 \mathrm{~Hz}$ (b) $14 \mathrm{~Hz}$ (c) white noise

\section{Conclusions}

\section{A On the Chebyshev polynomials}

A.1 Expression of the matrix of derivation

The matrix $\underline{\underline{D}}$, introduced in Eq. (5) to express the derivative of Chebyshev polynomials is given, in the case of even $p$, by

$$
\underline{\underline{D}}=\left(\begin{array}{ccccccccc}
0 & 0 & 0 & 0 & 0 & 0 & \cdots & 0 & 0 \\
1 & 0 & 0 & 0 & 0 & 0 & \cdots & 0 & 0 \\
0 & 4 & 0 & 0 & 0 & 0 & \cdots & 0 & 0 \\
3 & 0 & 6 & 0 & 0 & 0 & \cdots & 0 & 0 \\
0 & 8 & 0 & 8 & 0 & 0 & \cdots & 0 & 0 \\
5 & 0 & 10 & 0 & 10 & 0 & \cdots & 0 & 0 \\
\vdots & \vdots & \vdots & \vdots & \vdots & \vdots & \ddots & \vdots & \vdots \\
p-1 & 0 & 2(p-1) & 0 & 2(p-1) & 0 & \cdots & 0 & 0 \\
0 & 2 p & 0 & 2 p & 0 & 2 p & \cdots & 2 p & 0
\end{array}\right),
$$


and in the case of odd $p$, by

$$
\underline{\underline{D}}=\left(\begin{array}{ccccccccc}
0 & 0 & 0 & 0 & 0 & 0 & \cdots & 0 & 0 \\
1 & 0 & 0 & 0 & 0 & 0 & \cdots & 0 & 0 \\
0 & 4 & 0 & 0 & 0 & 0 & \cdots & 0 & 0 \\
3 & 0 & 6 & 0 & 0 & 0 & \cdots & 0 & 0 \\
0 & 8 & 0 & 8 & 0 & 0 & \cdots & 0 & 0 \\
5 & 0 & 10 & 0 & 10 & 0 & \cdots & 0 & 0 \\
\vdots & \vdots & \vdots & \vdots & \vdots & \vdots & \ddots & \vdots & \vdots \\
0 & 2(p-1) & 0 & 2(p-1) & 0 & 2(p-1) & \cdots & 0 & 0 \\
p & 0 & 2 p & 0 & 2 p & 0 & \cdots & 2 p & 0
\end{array}\right) .
$$

A.2 Coefficients of the expansion of a signal

Let $\check{u}$ be a function defined for $\tau \in[-1,1]$, which the values are known at $(m+1)$ points $-1=\tau_{0}<\tau_{1}<\cdots<\tau_{m}=1$. To estimate the coefficients of the expansion of $\check{u}$ on the Chebychev basis, this function is supposed to be piecewise affine :

$$
\forall i \in \llbracket 0, m-1 \rrbracket \quad \forall \tau \in\left[\tau_{i}, \tau_{i+1}\right] \quad \check{u}(\tau)=\check{u}\left(\tau_{i}\right)+\frac{\check{u}\left(\tau_{i+1}\right)-u\left(\tau_{i}\right)}{\tau_{i+1}-\tau_{i}}\left(\tau-\tau_{i}\right) .
$$

The coefficients of the expansion are then given by $\left\langle\check{u}, T_{s}\right\rangle /\left\langle T_{s}, T_{s}\right\rangle$, where

$$
\begin{aligned}
\left\langle\check{u}, T_{s}\right\rangle= & \int_{-1}^{1} \frac{\check{u}(\tau) \cos (s \arccos \tau)}{\sqrt{1-\tau^{2}}} d \tau, \\
= & \sum_{i=0}^{m-1}\left(\check{u}\left(\tau_{i}\right)-\frac{\check{u}\left(\tau_{i+1}\right)-\check{u}\left(\tau_{i}\right)}{\tau_{i+1}-\tau_{i}} \tau_{i}\right) \int_{\tau_{i}}^{\tau_{i+1}} \frac{\cos (s \arccos \tau)}{\sqrt{1-\tau^{2}}} d \tau \\
& \quad+\sum_{i=0}^{m-1} \frac{\check{u}\left(\tau_{i+1}\right)-\check{u}\left(\tau_{i}\right)}{\tau_{i+1}-\tau_{i}} \int_{\tau_{i}}^{\tau_{i+1}} \frac{\tau \cos (s \arccos \tau)}{\sqrt{1-\tau^{2}}} d \tau .
\end{aligned}
$$

The integrals of this expression are given by

$$
\begin{array}{rlrl}
\int_{\tau_{i}}^{\tau_{i+1}} \frac{\cos (s \arccos \tau)}{\sqrt{1-\tau^{2}}} d \tau= & {[\arcsin \tau]_{\tau_{i}}^{\tau_{i+1}}} & \text { if } s=0 \\
& -\frac{1}{s}[\sin (s \arccos \tau)]_{\tau_{i}}^{\tau_{i+1}} & & \forall s \geq 1 \\
\int_{\tau_{i}}^{\tau_{i+1}} \frac{\tau \cos (s \arccos \tau)}{\sqrt{1-\tau^{2}}} d \tau= & -[\sin (\arccos \tau)]_{\tau_{i}}^{\tau_{i+1}} & \text { if } s=0 \\
& -\frac{1}{4}[2 \arccos \tau+\sin (2 \arccos \tau)]_{\tau_{i}}^{\tau_{i+1}} & & \text { if } s=1 \\
& \frac{1}{s^{2}-1}[\sin (\arccos \tau) \cos (s \arccos \tau) & \\
-s \tau \sin (s \arccos \tau)]_{\tau_{i}}^{\tau_{i+1}} & \forall s \geq 2 .
\end{array}
$$

\section{References}

Adhikari S (2004) Optimal complex modes and index of damping non-proportionality. Mechanical systems and signal processing 18(1):1-27

Erlicher S, Argoul P (2007) Modal identification of linear non-proportionally damped systems by wavelet transform. Mechanical systems and signal processing 21(3):1386-1421 
Le TP, Argoul P (2004) Continuous wavelet transform for modal identification using free decay response. Journal of sound and vibration 277(1-2):73-100

Pacheco RP, Steffen VJ (2002) Using orthogonal functions for identification and sensitivity analysis of mechanical systems. Journal of Vibration and Control 8(7):993-1021

Rémond D, Neyrand J, Aridon G, Dufour R (2008) On the improved use of Chebyshev expansion for mechanical system identification. Mechanical Systems and Signal Processing 22:390-407

Slavic J, Simonovski I, Boltezar M (2003) Damping identification using a continuous wavelet transform: applicaton to real data 262:291-307

Staszewski W (1997) Identification of damping in mdof systems using time-scale decomposition 203(2):283-305

Staszewski W (1998) Identification of non linear systems using multi-scale ridges and skeletons of the wavelet transform 214(4):639-658 